\title{
Acid-based resorcinol-formaldehyde xerogels synthesized by microwave heating
}

Isabel D. Alonso-Buenaposada, Natalia Rey-Raap*, Esther G. Calvo, J. Angel Menéndez, Ana Arenillas

Instituto Nacional del Carbón CSIC, Apartado 73, 33080 Oviedo (Spain)

\begin{abstract}
The polymerization reaction that takes place between resorcinol and formaldehyde is spontaneous but slow. For this reason, compounds are often used to increase the reaction rate and reduce the synthesis time. These compounds can be basic or acidic and their nature and concentration can be used to modify the mechanisms of the reaction and the final properties of the materials. In this work the differences in the final properties of the organic xerogels obtained with basic or acid boosters have been studied. It was found that, irrespective of the nature of the booster, none of the end-product materials showed any differences in their chemical properties. Moreover, the concentrations of the components of the precursor solution (i.e. monomers, water and methanol) were observed to have the same effect on the porous properties of the materials regardless of whether an acidic or a basic booster was used. However, differences in the porous properties were observed. It was found that the methanol content was crucial to tailor the porosity over the entire nanoscale when an acidic booster is used. These results are of great importance as acidic boosters allows to decrease synthesis time and, hence, to produce more competitive materials.
\end{abstract}

Keywords: Organic xerogels, pH, microwave heating, porosity, resorcinol, formaldehyde

* nataliarey@fe.up.pt (ORCID: 0000-0002-5003-0035)

Telephone number: 00351220414931 


\section{Introduction}

Resorcinol-formaldehyde (RF) gels are porous materials obtained by means of a polymerization reaction [1-3]. These materials have aroused widespread interest among the scientific community over the past two decades due to their excellent properties and diverse applications [4-8,3]. Some of the most notable features of these materials include their high purity, low density, controllable porous structure and the possibility of doping them with metals or other substances [8-10,6]. Another reason for the growing interest in these polymeric materials has been the development of a simple and fast synthesis process based on microwave technology, which has led to a reduction in the synthesis time and manufacturing costs $[11,12]$. From this process, the so-called xerogels are obtained instead of the well-known aerogels, which are unfeasible to synthesize at an industrial scale [3]. Moreover, it has been widely demonstrated that microwave heating leads to xerogels with similar properties than those obtained by conventional methods [12]. Therefore, it is now possible for companies to take benefit from the advantageous characteristics of RF xerogels without incurring excessive production costs. However, as in all industrial processes, more research is needed to further reduce the synthesis time and, in turn, increase the competitiveness of the material produced. In the context of microwave-assisted synthesis, one of the possibilities to further minimize production time is to accelerate the chemical reaction that takes place during the synthesis process [13].

Generally, RF gels are synthesized by a sol-gel process via the polycondensation of resorcinol and formaldehyde in an aqueous solution [3]. Another compound, the socalled booster throughout this publication, is commonly used to regulate the $\mathrm{pH}$ of the precursor solution and accelerate the formation of the polymeric structure $[9,3,14,15]$. 
Usually, the compounds employed to promote the polymerization reaction between the resorcinol and formaldehyde are basic, being $\mathrm{Na}_{2} \mathrm{CO}_{3}$ and $\mathrm{NaOH}$ the most widely used bases [16-18,4,19]. However, some acid compounds such as perchloric, acetic, nitric or oxalic acids have also been employed in the synthesis of RF gels [20,17,21,4,22]. Some of these studies have demonstrated that acidic boosters lead to an increase in the reaction rate in comparison with basic boosters and, hence, the synthesis time is reduced $[21,17]$. Another factor to bear in mind is the incorporation of impurities in the carbon structure $[19,13,20,18]$. Calvo et al. recently shown that the most common basic boosters provide impurities, which cause corrosion and slagging in steel reactors operating at high temperatures [19]. These reactors are typically employed in the carbonization process of RF gels, which give rise to carbon gels. Therefore, the selection of an acid booster may contributed to avoid the incorporation of metals.

Nevertheless, despite the abovementioned advantages, the vast amount of literature on basic boosters overshadows the few reports focused on acidic boosters (the reader is referred to some of these relevant publications on acid-based RF gels $[1,20,17,13,15,23])$. From those studies, two main items should be highlighted: i) almost all acid-based RF gels synthesized are mainly macroporous materials but with lower densities than that of basic-based gels, and ii) from the best of our knowledge, all the studies reported to date, are based on the synthesis of aerogels rather than xerogels, the latter being those currently synthesized at an industrial scale by means of microwave heating. Consequently, although several attempts to synthesized acid-based aerogels have been made, there is still an ongoing need for a method for producing RF xerogels using acidic boosters, which gives rise to materials with tailored porosity over the entire nanoscale.

Therefore, the aim of the present work is to synthesize acid-based RF xerogels by 
means of microwave heating in order to i) obtain materials with tailored porosity and ii) increase the competitiveness of these materials by reducing synthesis time. It is wellknown that the porosity of basic-based RF xerogels can be minutely tuned by selecting the appropriate composition of the precursor solution: $\mathrm{pH}$, dilution ratio, $\mathrm{R} / \mathrm{F}$ molar ratio and even, as recently demonstrated, the methanol content in commercial formaldehyde solutions $[24,22,25,26,14,27,28]$. However, from the best of our knowledge, there are not exhaustive studies on the effect of all these variables on the porous properties of organic xerogels synthetized by employing acid as booster and a microwave oven as heating source. Accordingly, in this work the influence of the aforementioned variables on the chemical and porous properties of acid-based RF xerogels synthesized by microwave heating has been studied. All of the synthesized xerogels were characterized from the point of view of their chemical and porous structure to determine the effect of each variable. Moreover, basic-based RF xerogels have also been synthesized to ascertain possible differences in properties due to the booster employed.

\section{Experimental Section}

\subsection{Synthesis of organic xerogels}

A wide spectrum of resorcinol-formaldehyde xerogels was synthesized by microwave heating and by modifying chemical variables such as the $\mathrm{pH}$ of the precursor solution, the amount of solvent (water), the $\mathrm{R} / \mathrm{F}$ molar ratio and the amount of methanol contained in the commercial formaldehyde solution. Briefly, the synthesis of the organic xerogels proceeded as follows: a mixture of resorcinol (Indspec, 99.6 wt. \%), distilled water, formaldehyde (Química S.A.U., aqueous solution with 37 wt. \% formaldehyde

and 1.5 wt. \% methanol), and some extra methanol (AnalaR Normapur, 99\%) if necessary, was prepared. The initial $\mathrm{pH}$ of the precursor solution, which had a value of 
ca. 3.0 in almost all cases, was measured and then adjusted to the desired final $\mathrm{pH}$ in order to obtain acid-based and basic-based xerogels. To this end, nitric acid (Merck, 65 wt. $\% \mathrm{HNO}_{3}$ ) and sodium hydroxide $(1 \mathrm{M} \mathrm{NaOH}$ made up of solid $\mathrm{NaOH}$, AnalaR Normapur, $99.9 \%$ ) were used as boosters to adjust the $\mathrm{pH}$ of the precursor solutions, respectively.

Resorcinol, formaldehyde, methanol and water were added in the required proportions depending on the values of dilution ratio $(\mathrm{D}=$ total solvent $/$ reactant molar ratio), R/F molar ratio and percentage of methanol desired. These values were selected on the basis of results reported in previous published works on the synthesis of basic-based RF xerogels, in order to obtain materials with different porous properties $[27,14]$.

Each precursor solution prepared was introduced into the microwave oven at $85{ }^{\circ} \mathrm{C}$ for about 5 hours, following the experimental method describe elsewhere [11]. Once the synthesis was completed, the organic xerogels were subjected to a heat treatment at 100 ${ }^{\circ} \mathrm{C}$ overnight to remove any traces of reagent and obtain completely stable samples.

The nomenclature of each sample indicates the synthesis conditions employed; OXA and OXB denoting Organic Xerogel prepared with an Acid booster and Organic Xerogel prepared with a Basic booster, respectively, and the following numbers referring to the $\mathrm{pH}$, dilution ratio, $\mathrm{R} / \mathrm{F}$ molar ratio and the percentage of methanol content, in that order.

\subsection{Characterization techniques}

The chemical composition of the RF xerogels was evaluated in order to identify possible discrepancies between the samples synthesized via the acid and basic routes. Therefore, ultimate (i.e. carbon, hydrogen, nitrogen and oxygen contents) and proximate 
(i.e. moisture, ash and volatile matter contents) measurements were carried out in a LECO-TF-900 and LECO-CHNS-932 microanalyzer, respectively.

Fourier Transform Infrared Spectroscopy (FTIR) was also performed. The spectra were recorded in the 525 and $4000 \mathrm{~cm}^{-1}$ range on a Nicolet IR 8700 spectrometer fitted with a DTGS detector (deuterated triglycine sulphate) at a resolution of $4 \mathrm{~cm}^{-1}$ over 64 accumulated scans. All the samples were analyzed twice. In order to prepare the pellets, the samples and $\mathrm{KBr}$ (previously dried overnight) were mixed in a proportion of 1:100 in an agate mortar for 10 minutes until a homogeneous mixture was obtained. Around $0.125 \mathrm{~g}$ of this mixture was subjected to 8 tons of pressure in a $13 \mathrm{~mm}$-diameter matrix.

In addition, in order to study the chemical properties of the xerogels in greater depth the $\mathrm{pH}$ of the point of zero charge $\left(\mathrm{pH}_{\mathrm{PZC}}\right)$ was determined by the reverse mass titration method described elsewhere [29].

The porous structure of the RF xerogels was evaluated by the following techniques: (i) nitrogen adsorption-desorption isotherm analysis and; (ii) mercury porosimetry. Prior to performing these analyses, the samples were degassed at $120^{\circ} \mathrm{C}$ under a vacuum of 0.1 mbar overnight. $\mathrm{N}_{2}$ adsorption-desorption isotherms were performed at $-196^{\circ} \mathrm{C}$ using a Tristar II 3020 (Micromeritics) analyzer. The specific surface area was calculated by the Brunauer-Emmett-Teller (BET) method. The Dubinin-Raduskevich (D-R) model was used to obtain information about the microporosity ( $\left.\mathrm{V}_{\mathrm{DUB}-\mathrm{N} 2}\right)$. The total pore volume $\left(\mathrm{V}_{\mathrm{p}}\right)$ was calculated from the amount of nitrogen adsorbed at a relative pressure of 0.99 . The mesopore volume obtained by this technique $\left(\mathrm{V}_{\text {meso-N2 }}\right)$ was calculated as the difference between $V_{p}$ and the $V_{\text {DUB-N2. Pore volume measurements by nitrogen }}$ adsorption are not precise enough for samples containing macropores. As several of the studied samples displayed large mesopores, and in some cases macropores, mercury 
porosimetry was used as a complementary technique. The device used was an AutoPore IV 9500 Micromeritics that is able to operate in a pressure range from atmospheric pressure to $228 \mathrm{MPa}$. The surface tension and contact angle values for $\mathrm{Hg}$ in all the characterizations were $0.485 \mathrm{~N} \mathrm{~m}^{-1}$ and $130^{\circ}$, respectively. By means of this technique, based on the Washburn intrusion theory, the following parameters were calculated: mesopore and macropore volume $\left(\mathrm{V}_{\text {meso }}\right.$ and $\mathrm{V}_{\text {macro, }}$, respectively), average pore size $\left(\mathrm{d}_{\text {pore }}\right)$, percentage of porosity and bulk density. It should be noted that the lowest detectable limit of the device is $5.5 \mathrm{~nm}$, so that, $\mathrm{V}_{\text {meso }}$ refers to a pore size range of between 50 and $5.5 \mathrm{~nm}$. Moreover, it should be highlight that some organic gels may have a weak compressive strength and they may undergo densification without any intrusion of mercury into the pores when submitted to mercury porosimetry. In order to avoid possible errors due to densification, the analysis of the data obtained from mercury porosimetry was treated following the methodology detailed elsewhere [30].

The morphology of all samples was examined using a Quanta FEG 650 scanning electron microscope. Samples were previously attached to an aluminum tap using conductive double-sided adhesive tape. An accelerating voltage of $25 \mathrm{kV}$ and a secondary electron detector EDT (Everhart-Thornley) were used in all analysis.

\section{Results and Discussion}

\subsection{Chemical properties}

The chemical composition of acid-based (OXA) and basic-based (OXB) RF xerogels synthesized was analysed. Given that, as expected, the composition of all materials studied was similar, only the results of four samples are shown in Table 1, as an example. 
Table 1. Elemental analysis of the acid and basic RF xerogels.

\begin{tabular}{c|cc|cc}
\hline Booster & \multicolumn{2}{|c|}{ Acid $\left(\mathrm{HNO}_{3}\right)$} & \multicolumn{2}{c}{ Basic $(\mathrm{NaOH})$} \\
\hline Sample & OXA-2.0-3.7-0.5-12.5 & OXA-2.0-2.7-0.5-1.5 & OXB-5.4-7.7-0.3-12.5 & OXB-6.5-6.0-0.5-12.5 \\
\hline $\mathrm{C}( \pm 0.3$, wt. \%) & 65.8 & 66.1 & 64.7 & 66.3 \\
$\mathrm{H}( \pm 0.3$, wt. \%) & 4.3 & 4.6 & 4.9 & 4.5 \\
$\mathrm{~N}( \pm 0.3$, wt. \%) & 0.3 & 0.3 & 0.3 & 0.3 \\
$\mathrm{O}( \pm 0.3$, wt. \%) & 29.6 & 29.0 & 30.1 & 28.9 \\
\hline
\end{tabular}

All samples contain c.a. 65.5 wt. $\%$ of carbon, 4.5 wt. $\%$ of hydrogen and 30 wt. $\%$ of oxygen. No nitrogen is considered to be present since $0.3 \mathrm{wt} . \%$ is within the range of analysis error and the precursors (i.e., $\mathrm{R}$ and F) have no nitrogen. It should also be noted that the same nitrogen content was obtained for all the samples which means that the nitrogen content did not increase (at least substantially) as a result of using $\mathrm{HNO}_{3}$ for the synthesis. Moreover, all the samples showed c.a. $46 \%$ of volatile material, $5 \%$ of moisture and no mineral residue at all. Therefore, irrespective of the nature of the booster employed to fix the $\mathrm{pH}$, the chemical composition obtained was similar for all samples, inasmuch as the same main reagents were used to prepare the precursor solutions. However, it is well-known that the nature of the booster modifies the mechanism of the polymeric reaction [10,3]. Accordingly, structural differences may be expected between OXA and OXB samples. Conversely, as shown in Figure 1, the FTIR spectra of acid (series OXA) and basic (series OXB) samples are quite similar (note that only four samples are shown in Figure 1, as an example). These results are in agreement with those recently published by Alonso-Buenaposada et al. [28]. The authors presented an exhaustive chemical study on organic and carbon basic-based xerogels synthesized by microwave heating in which several techniques of characterization were employed to elucidate the possible differences in the chemical structure of those materials [28]. They demonstrated that all samples present a very similar chemical properties, even slightly 
differences related to the concentration of oxygen and number of ester bonds were found [28].

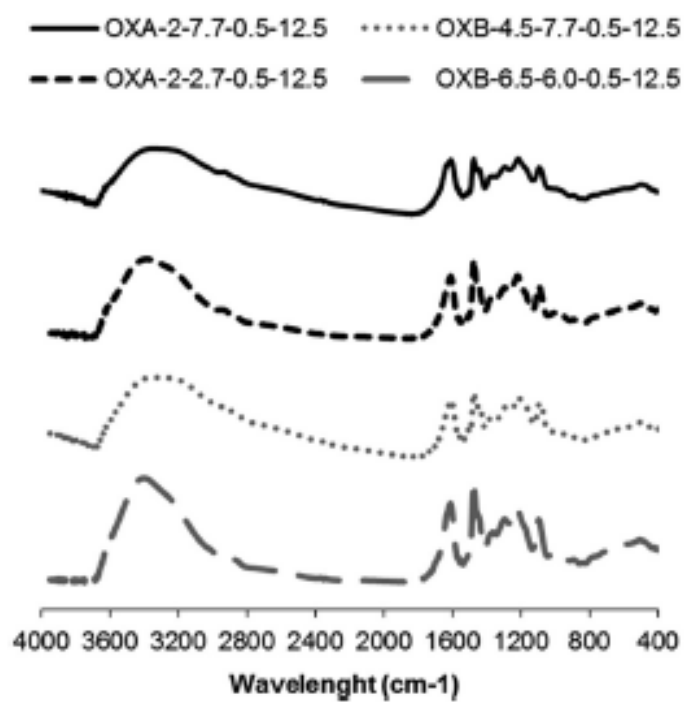

Fig. 1. FTIR spectra of the RF xerogels synthesized by the addition of an acid $\left(\mathrm{HNO}_{3}\right)$ or basic $(\mathrm{NaOH})$ booster

As shown in Figure 1, all the samples exhibit a wide band between 3700 and $3000 \mathrm{~cm}^{-1}$, associated with $\mathrm{O}-\mathrm{H}$ stretching vibrations, while another band located at $1474 \mathrm{~cm}^{-1}$, corresponds to $\mathrm{CH}_{2}$ deformation vibrations [5]. Moreover, the band at $1613 \mathrm{~cm}^{-1}$ is due to aromatic ring stretching vibrations $(\mathrm{C}=\mathrm{C})$ whereas the bands at 1217 and $1092 \mathrm{~cm}^{-1}$ indicate the presence of methylene ether bridges C-O-C. These similarities found in the chemical properties of the RF xerogels synthesized from precursor solutions with boosters of different nature can be attributed to the two steps involved in the polymerization reaction between resorcinol and formaldehyde: (i) addition and (ii) condensation reaction [2]. In general, the mechanism of the addition reaction depends largely on the nature of the compound used to adjust the $\mathrm{pH}$ of the precursor solution, whilst the condensation reaction is independent of the booster, as shown in Figure 2. 
a) Basic route

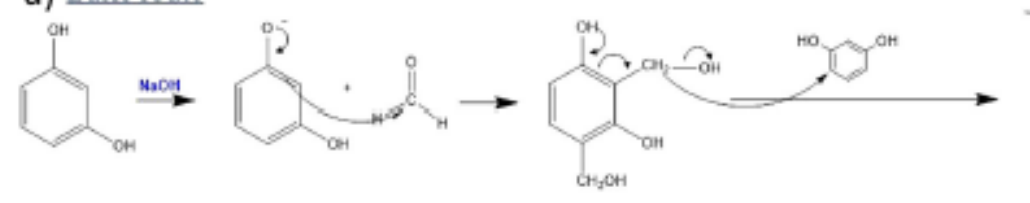

b) Acid route
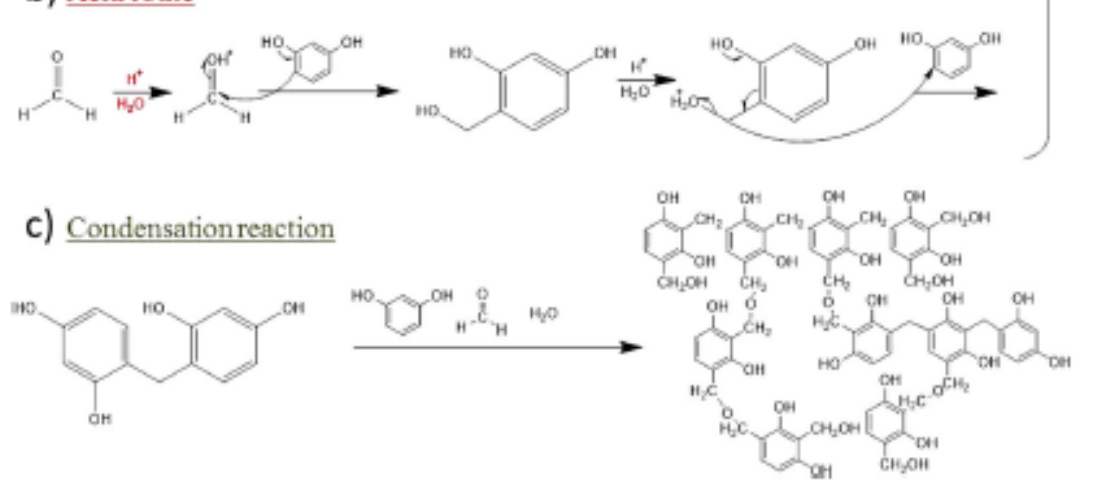

Fig. 2. Mechanism of the polymerization reaction between resorcinol and formaldehyde under basic and acid routes

When a basic booster is used, the resorcinol is deprotonated (Figure 2a), giving rise to resorcinol anions which trigger a nucleophilic attack from the second, fourth or sixth positions of the resorcinol towards the formaldehyde [2,25,3,23]. On the other hand, when an acid compound is used, the first step is favoured by the protonation of the formaldehyde (Figure 2b), which makes the carbonyl carbon more electrophilic and causes an identical $\mathrm{SN}_{2}$ attack from the resorcinol [1]. As a result of these two mechanisms, hydroxymethyl derivatives are obtained, which are the necessary monomers for polymerization to occur. A series of consecutive condensation reactions then take place, in which the hydroxymethyl derivatives lose $\mathrm{OH}$ groups and form benzyl-type cations $[26,15,23]$. Each cation reacts with a benzene ring of another molecule, giving rise to methylene and ether bonds until a three-dimensional crosslinked polymer is formed, as shown in Figure 2c. The results of FTIR shown in Figure 1 , are in good agreement with the above detailed mechanisms, as all the materials have similar chemical properties, whichever booster was used. 


\subsection{Porous properties}

The most interesting characteristic of basic-based RF xerogels is that it is possible to design their porous properties to fit the requirement of specific applications, by modifying the main chemical variables: $\mathrm{pH}, \mathrm{D}, \mathrm{R} / \mathrm{F}$ molar ratio and $\%$ of methanol. The effect of the $\mathrm{pH}$ is the most studied variable $[17,12,14,9]$. Values of $\mathrm{pH}$ usually ranged between 3.0, which is the initial $\mathrm{pH}$ value of most of the precursor solutions, and 7.0 $[12,26]$. Generally, an increase in the concentration of the basic booster, i.e. an increase in the $\mathrm{pH}$ value, results in materials with smaller pore size, which is confirmed by pore size distributions shown in Figure 3a. Therefore, one might assume an increase in pore size as the $\mathrm{pH}$ drops below 3.0. However, contrary to expected, pore size of samples synthesized with an acidic booster decreases with $\mathrm{pH}$, as shown in Figure $3 \mathrm{~b}$. It is evident from these results that the polymerization reaction depends largely on the amount and nature of catalyst added. Each booster favours its corresponding addition reaction (Figure $2 \mathrm{a}$ and $2 \mathrm{~b}$ ), which leads to the formation of a large number of clusters and, hence, to materials with pores of smaller size. However, it should be noted that the use of a basic or an acidic booster results in meso-macroporous materials or exclusively macroporous materials, respectively. These results are consistent with other published studies where it was shown that the use of acidic boosters gives rise to materials of larger pore size [13]. Therefore, it seems necessary to modify the concentration of the remaining reagents (i.e. $\mathrm{R} / \mathrm{F}$ molar ratio, $\mathrm{D}$ and percentage of methanol) to find out if it is possible to obtain porous materials over the entire nanoscale by using acidic boosters. 

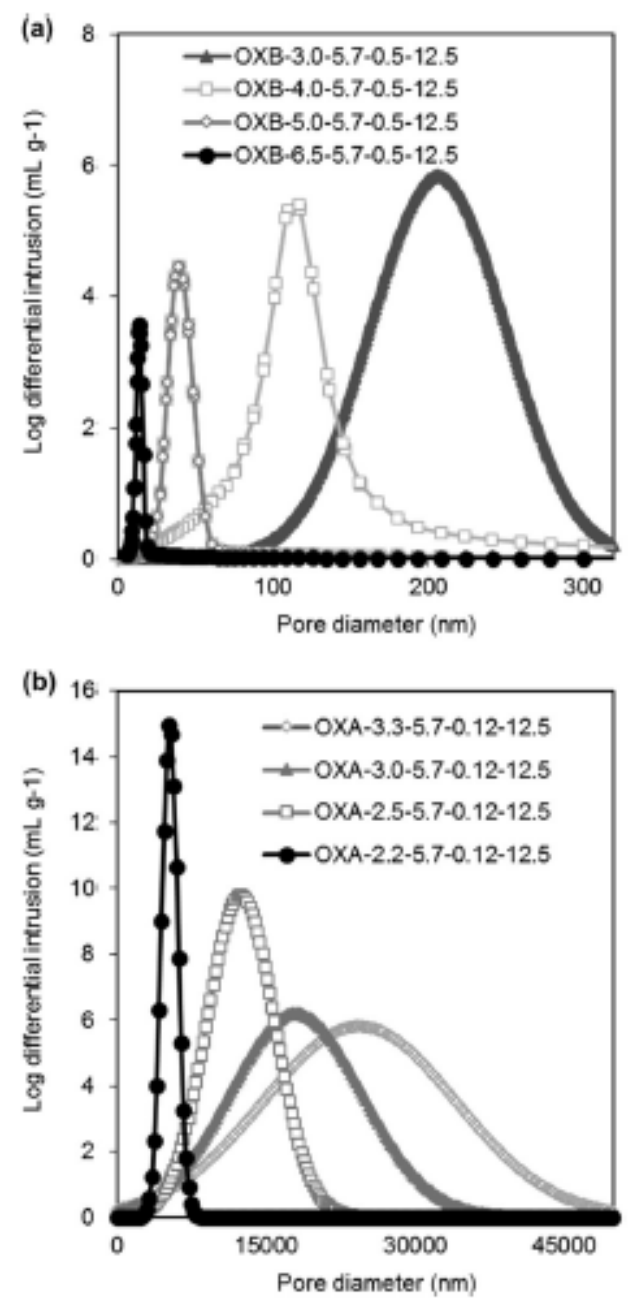

Fig. 3. Influence of the $\mathrm{pH}$ value on the pore size distribution of RF xerogels synthesized by adding basic (a) or acid (b) boosters

Initially, two set of samples (OXB and OXA) were synthesized from precursor solutions with different $\mathrm{R} / \mathrm{F}$ molar ratio. The effect of this variable on pore size and total pore volume obtained by mercury porosimetry, is shown in Figure 4. Regardless of the nature of the booster, a decrease in the concentration of either of the main reagents (resorcinol and formaldehyde) leads to a decrease in the reaction rate since the precursors are prevented from locating each other. As a consequence, an increase in the pore size is observed, and a minimum value of pore size is obtained when the stoichiometric ratio $(\mathrm{R} / \mathrm{F}=0.5)$ is employed. On the other hand, an excess of 
formaldehyde (i.e., a low R/F molar ratio) creates more interconnections between clusters, leading to a highly branched structure $[22,14]$. The mechanical strength of these RF xerogels is greater so that no shrinkage occurs during the drying step, which results in a larger total pore volume (Figure 4). Therefore, whereas same pore size can be obtained using different $\mathrm{R} / \mathrm{F}$ molar ratios, large pore volumes are achieved only if the $\mathrm{R} / \mathrm{F}$ molar ratio is below 0.5 . The effect of modifying the $\mathrm{R} / \mathrm{F}$ molar ratio is similar for both the OXA and OXB series. However, the porous properties of samples synthesized by using an acidic booster are within the range of macroporosity, while OXB are within the range of mesoporosity. This effect, as explained above, is due to the amount and nature of the booster, which means that $\mathrm{pH}$ value has more effect on the porous properties than the $\mathrm{R} / \mathrm{F}$ molar ratio.
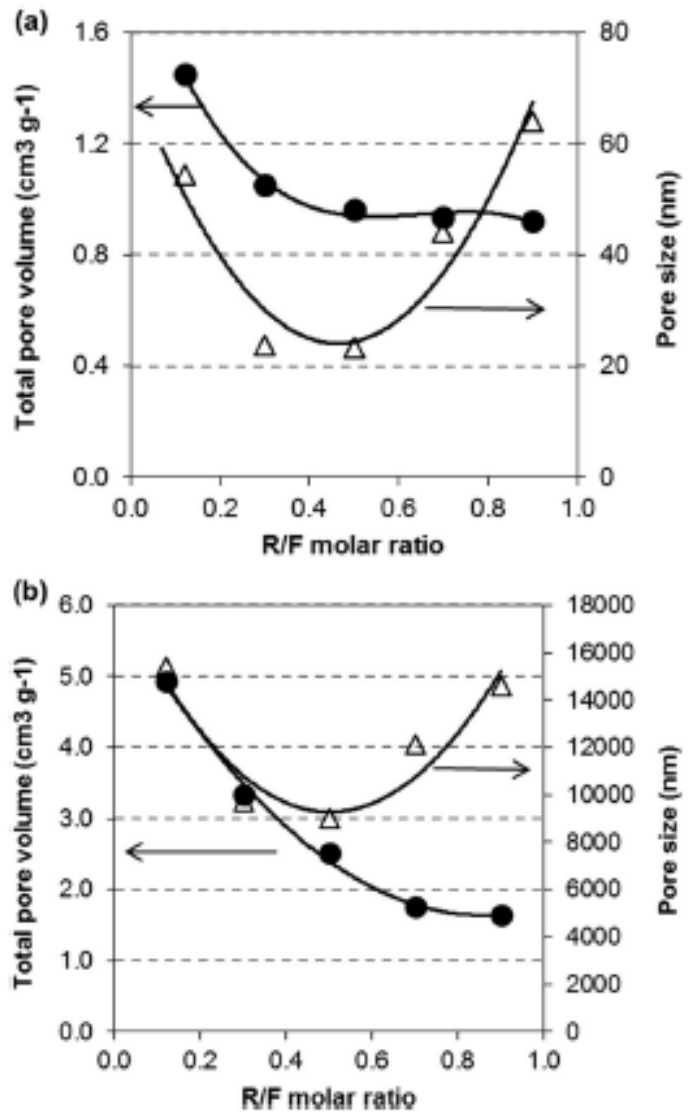

Fig. 4. Effect of the variation of the R/F molar ratio on the pore size $(\Delta)$ and total 
pore volume ( $\bullet$ ) when (a) a basic $(\mathrm{NaOH})$ or (b) an acid (HNO3) booster is used to prepare the precursor solution

Another important chemical variable which must be taken into account when designing the porosity of RF xerogels is the dilution ratio (D). In order to evaluate the effect of this parameter, the pore size distribution obtained by subjecting the samples with different dilution ratios to mercury porosimetry is shown in Figure 5. The same trend can be observed by both the OXA and OXB series: the larger the dilution ratio is, the larger the pore size obtained. This is because an increase in the dilution ratio leads to a greater volume of water among the clusters, which in turn, results in an increase in the distance between clusters, and therefore, larger pores [24,26]. As in the case of basic compounds, the OXA series shows an upper limit for D values and, when it is exceeded, the sol-gel reaction fails to take place as the large amount of water prevents the precursor solution from reaching its gelation point [26]. 

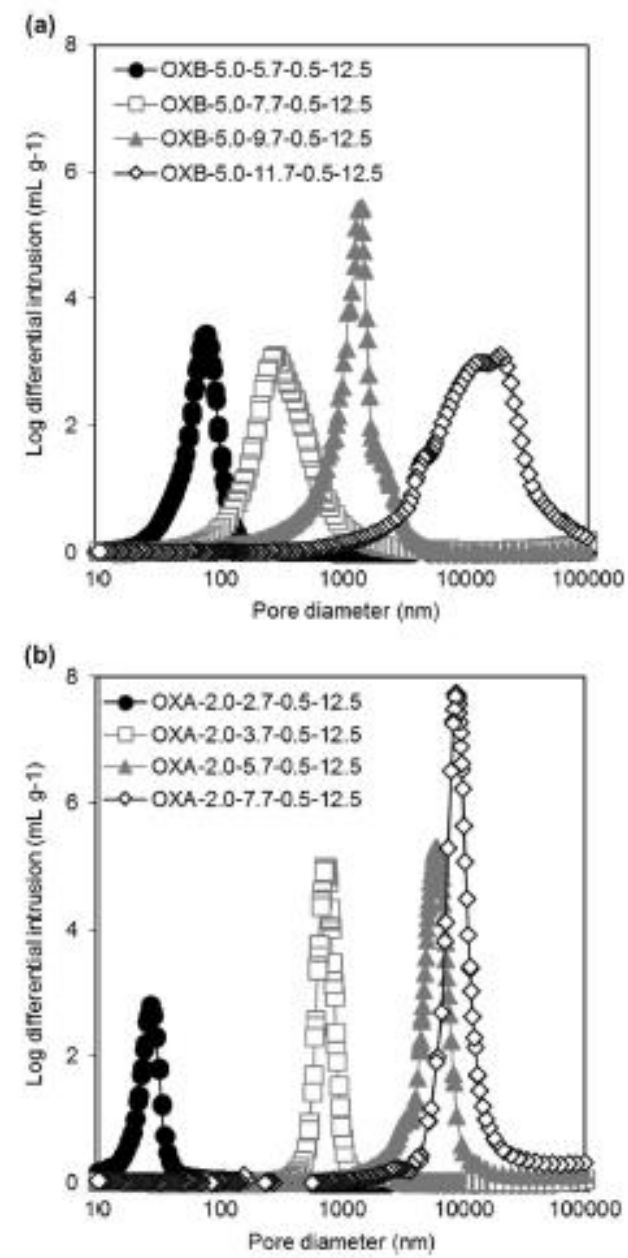

Fig. 5. Influence of the dilution ratio on the pore size distribution of RF xerogels synthesized by adding basic (a) or acid (b) boosters

Finally, the influence of the concentration of methanol on the porous properties of RF xerogels prepared from acidic and basic precursor solutions was evaluated. Methanol is added to formaldehyde solutions in order to prevent the formaldehyde from polymerizing and subsequently precipitating. It was only recently that the methanol content of the formaldehyde solution was recognised as a chemical variable that affected the porosity of RF xerogels [27]. Hemiacetals and acetals are formed in a reversible reaction that prevents the formation of clusters owing to the fact that the reaction between resorcinol and formaldehyde only takes place if the formaldehyde remains in its free form [3]. Consequently, an increase in the methanol content leads to 
an increase in the formation of hemiacetals which prevents the resorcinol molecules from finding free formaldehyde molecules to react with. Moreover, the formation of hemiacetal compounds is favoured in acidic media [27], which suggest that the formation, growth and crosslinking of the polymeric structure depend to a great extent on the methanol content and the booster used to prepare the precursor solution. In fact, structural differences were observed by SEM images, as shown Figure 6. In Figure 6a and $6 \mathrm{~b}$ it can be appreciated the characteristic morphology of basic-based RF xerogels. However, by using an acidic booster, the typical morphology is replaced by a capsulelike or cellular morphology with pores enclosed by thin polymeric walls.
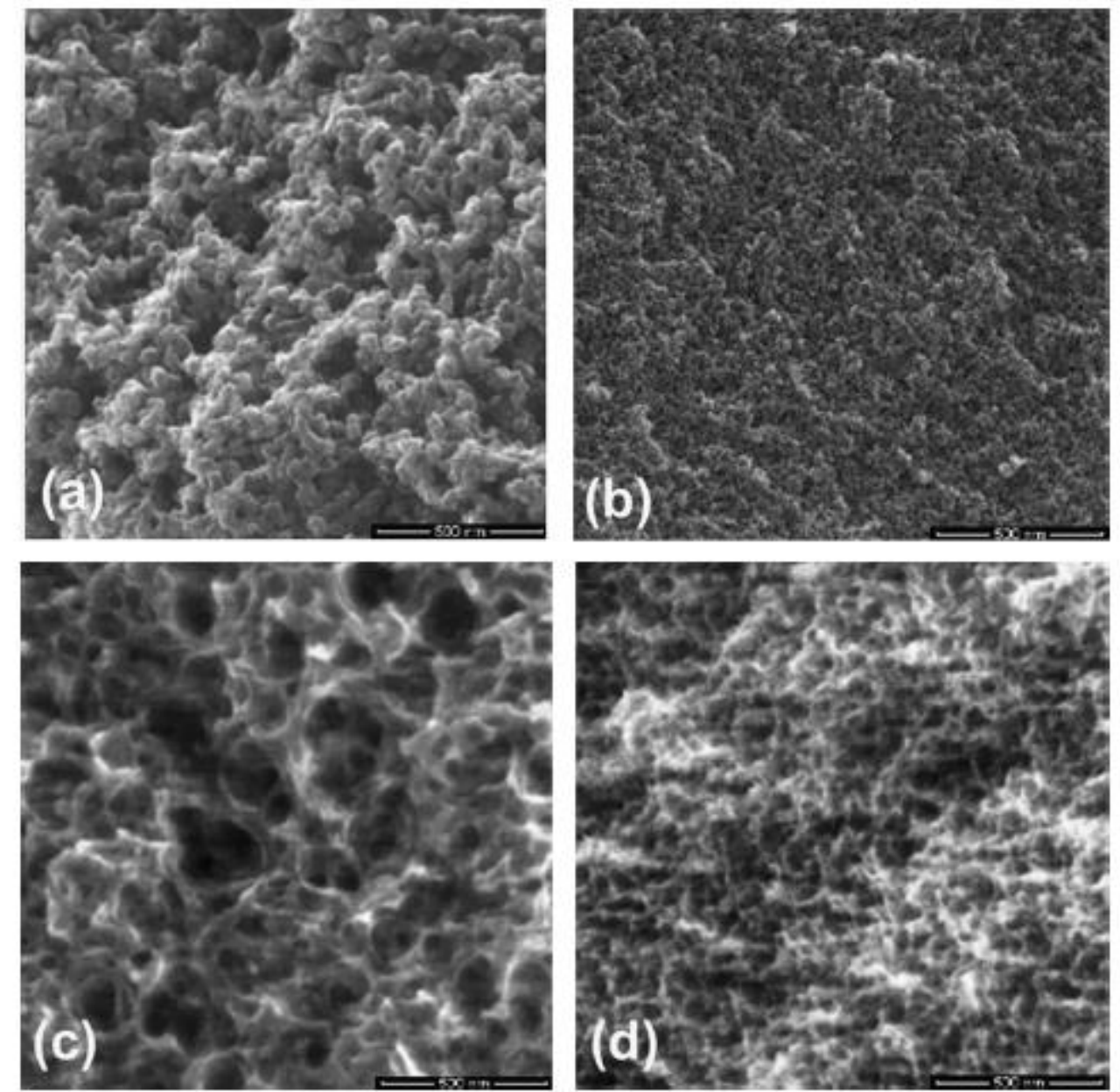

Fig. 6. SEM images of RF xerogels synthesized from precursor solutions with different concentration of reagents: (a) OXB-5.4-7.7-0.3-12.5, (b) OXB-6.5-6.0-0.512.5, (c) OXA-2.0-2.7-0.5-1.5 and (d) OXA-2.0-2.7-0.5-20.0 
However, even though differences in the polymeric structures have been found, the effect of methanol content on the reactivity of resorcinol (or the availability to polymerize) is independent of the nature of the booster used (acidic or basic). Therefore, similar effects on the final porous properties are observed, as shown in Figure 7. Materials synthesized with low concentrations of methanol have larger pore volumes both in the OXA and OXB series of samples.

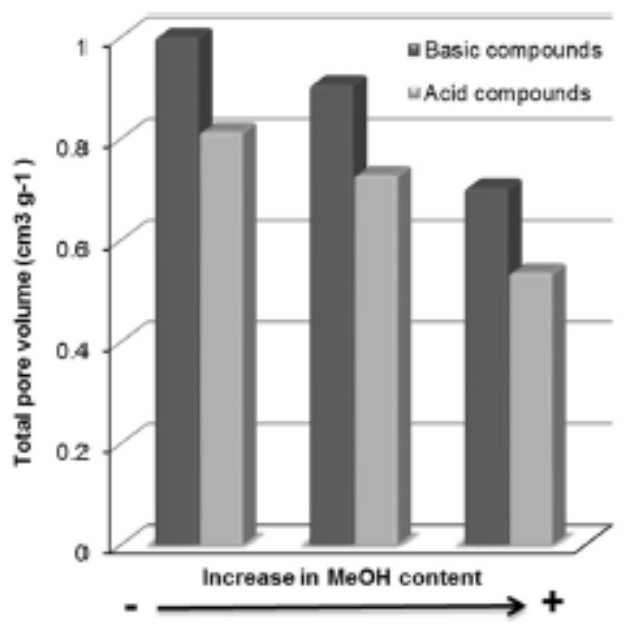

Fig. 7. Influence of the methanol content of the formaldehyde solutions on the porosity of RF xerogels synthesized using a basic (NaOH) or an acid (HNO3) booster in the precursor solution

As explained in detail in previous studies [27,10,14,26], the formation, growth and crosslinking of the polymeric structure depend on the concentrations of all the reagents used to prepare the precursor solution. Accordingly, it is possible to obtain RF xerogels with a desired pore size whether an acid or basic booster is used, if the concentration of all the reagents are properly selected, as shown in Table 2 . However, it is worth noting that the variation of the percentage of methanol in OXA series, results in mesoporous materials, which are generally achieved only by using basic boosters. In other words, the variation of the methanol content is essential in the case of acid-based RF xerogels 
in order to obtain mesoporous materials.

Table 2. The porous properties of the RF xerogels synthesized under different chemical conditions

\begin{tabular}{|c|c|c|c|c|c|c|c|c|}
\hline SAMPLE & $\begin{array}{c}{ }^{\mathrm{a}} \text { Vtot } \\
\left(\mathrm{cm}^{3} \mathrm{~g}^{-1}\right)\end{array}$ & $\begin{array}{l}{ }^{\mathrm{a}} \text { Vmeso } \\
\left(\mathrm{cm}^{3} \mathrm{~g}^{-1}\right)\end{array}$ & $\begin{array}{l}{ }^{\mathrm{a}} \text { Vmacro } \\
\left(\mathrm{cm}^{3} \mathrm{~g}^{-1}\right)\end{array}$ & $\begin{array}{c}\begin{array}{c}{ }^{\text {a Pore }} \\
\text { diameter } \\
(\mathbf{n m})\end{array} \\
\end{array}$ & $\begin{array}{l}{ }^{\mathrm{a} B u l k} \\
\text { density } \\
\left(\mathrm{g} \mathrm{cm}^{-3}\right)\end{array}$ & $\begin{array}{c}{ }^{\mathrm{a}} \text { Porosity } \\
(\%)\end{array}$ & $\begin{array}{l}{ }^{\mathrm{b}} \mathbf{S}_{\mathrm{BET}} \\
\left(\mathbf{m}^{2} \mathbf{g}^{1}\right)\end{array}$ & $\begin{array}{l}{ }^{b} V_{m e s o-N 2} \\
\left(\mathrm{~cm}^{3} \mathbf{g}^{-1}\right)\end{array}$ \\
\hline OXB-4.5-7.7-0.5-12.5 & 2.67 & 0.03 & 2.64 & 775 & 0.2 & 86 & 120 & 0.05 \\
\hline OXB-5.4-7.7-0.3-12.5 & 1.47 & 0.81 & 0.65 & 41 & 0.44 & 65 & 180 & 0.36 \\
\hline OXB-6.2-8.6-0.5-12.5 & 0.64 & 0.51 & 0.13 & 22 & 0.61 & 53 & 269 & 0.47 \\
\hline OXB-6.5-6.0-0.5-12.5 & 0.41 & 0.36 & 0.04 & 14 & 0.76 & 40 & 303 & 0.52 \\
\hline OXA-2.0-3.7-0.5-12.5 & 1.01 & 0 & 1.01 & 735 & 0.56 & 56 & 0 & 0 \\
\hline OXA-2.0-2.7-0.5-1.5 & 0.81 & 0.65 & 0.17 & 41 & 0.63 & 50 & 110 & 0.33 \\
\hline OXA-2.0-2.7-0.5-12.5 & 0.73 & 0.67 & 0.06 & 26 & 0.67 & 49 & 226 & 0.66 \\
\hline OXA-2.0-2.7-0.5-20.0 & 0.54 & 0.48 & 0.06 & 14 & 0.70 & 37 & 354 & 0.73 \\
\hline
\end{tabular}

In short, an appropriate combination of $\mathrm{pH}, \mathrm{R} / \mathrm{F}$ molar ratio, dilution ratio and methanol content leads to materials with similar porous properties irrespective of the mechanism of the polymerisation reaction (Figure 2). However, acidic boosters, should result in lower synthesis time and, hence, in to more competitive materials. In order to verify this advantage, the evolution of the $\mathrm{pH}$ of 4 precursor solutions was registered at ambient temperature and plotted in Figure 8.

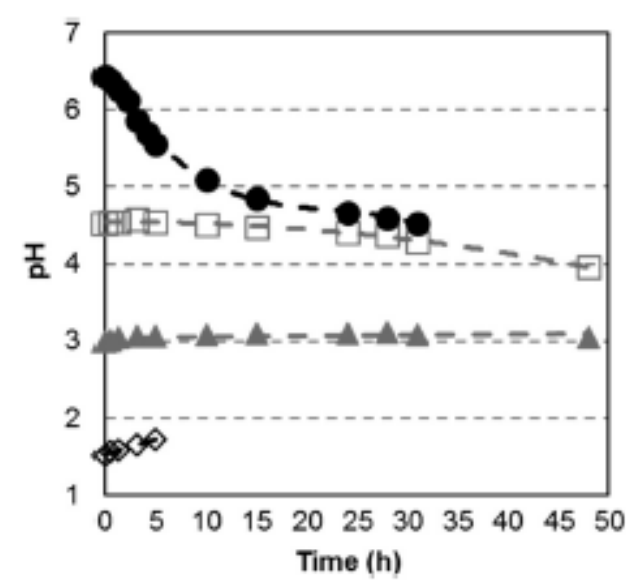

Fig. 8. Evolution of the $\mathrm{pH}$ of the precursor solutions with time up to the point of gelation for the following initials pH: $1.5,3$ (with no compound to modify the pH), 


\section{5 and 6.5}

From Figure 8 it can be inferred that, even at room temperature, the precursor solution reacts and its $\mathrm{pH}$ value changes with time. Moreover, although each solution has a different initial $\mathrm{pH}$, the general trend is for all of them to acquire the same final $\mathrm{pH}$ (c.a. $\mathrm{pH}$ of 3), which can be considered as the equilibrium $\mathrm{pH}$ for this type of materials. This $\mathrm{pH}$ value matches the $\mathrm{pH}_{\mathrm{PZC}}$ of all the final $\mathrm{RF}$ xerogels obtained (i.e. $\mathrm{pH}_{\mathrm{PZC}}=3$ for all organic xerogels), indicating that, although the type and concentration of the booster may have a great effect on the mechanism and rate of the polymerisation reaction, respectively, the final nature of the material obtained is similar. It can also be observed that the acidic solutions get to the gelation point faster than the basic ones even if they are farther from the equilibrium $\mathrm{pH}$ (e.g., the precursor mixture with a $\mathrm{pH}=1.5$ only shows a few points in Figure 8). In fact, a solution with a $\mathrm{pH}$ of 0.85 was prepared and no data at all could be recorded owing to its instant gelation. Therefore, the main difference between what an acid or a basic booster has to offer is that the acid mechanism (Figure $2 b$ ) is more favoured and so the reaction is much faster than when a basic one is used. Extra evidence for this is provided in the video of the supplementary material.

\section{Conclusions}

In this work resorcinol-formaldehyde (RF) xerogels have been synthesized by means of microwave heating and by using basic and acidic booster to adjust the $\mathrm{pH}$ of the precursor solutions. It was found that, regardless of the booster used, materials with similar chemical composition were obtained. Conversely, the nature of the booster had a great influence on the porous properties. It has been observed that basic boosters led to meso-macroporous materials while acid boosters resulted in exclusively macroporous 
materials. However, it has been demonstrated that the properties of acid-base RF xerogels can be also tailored by the appropriate selection of the concentration of all reagents, which are defined by the $\mathrm{R} / \mathrm{F}$ molar ratio, the dilution ratio and the methanol content. Among these three variables, the methanol content has shown to be crucial to obtained mesoporous materials via an acid route. These results are of great relevance as they proof, for the first time, that the microwave-assisted synthesis of basic-based organic xerogels may lead to materials with tailored porosity over the entire nanoscale. Moreover, acid boosters has the advantage that it prevents the incorporation of impurities into the carbonaceous structure (i.e. alkaline metals) and accelerates the polymerization reaction, leading also to more competitive materials.

\section{Acknowledgements}

The authors gratefully acknowledge the financial support of the Ministerio de Economía y Competitividad of Spain, MINECO (Project CTQ2014-54772-P).

\section{Bibliography}

1. Aegerter MA, Leventis N, Koebel MM (2011) Aerogels handbook. Advances in Sol-Gel Derived Materials and Technologies. Springer,

2. Al-Muhtaseb SA, Ritter JA (2003) Preparation and properties of resorcinol-formaldehyde organic and carbon gels. Adv Mater 15:101-114.

3. Rey-Raap N, Arenillas A, Menéndez JA (2015) Formaldehyde in the Synthesis of ResorcinolFormaldehyde Carbon Gels In: Patton A (ed) Formaldehyde: Synthesis, Applications and Potential Health Effects. Nova Science,

4. Alegre C, Sebastián D, Gálvez ME, Moliner R, Lázaro MJ (2016) Sulfurized carbon xerogels as Pt support with enhanced activity for fuel cell applications. Appl Catal, B 192:260-267.

5. Álvarez S, Ribeiro RS, Gomes HT, Sotelo JL, García J (2015) Synthesis of carbon xerogels and their application in adsorption studies of caffeine and diclofenac as emerging contaminants. Chem Eng Res Des 95:229-238.

6. Moreno-Castilla C, Maldonado-Hódar FJ (2005) Carbon aerogels for catalysis applications: An overview. Carbon 43:455-465.

7. Rey-Raap N, Piedboeuf MLC, Arenillas A, Menendez JA, Leonard AF, Job N (2016) Aqueous and organic inks of carbon xerogels as models for studying the role of porosity in lithium-ion battery electrodes. Materials \& Design 109:282-288.

8. Lu X, Shen J, Ma H, Yan B, Li Z, Shi M, Ye M (2012) A cost-effective way to maintain metal-doped carbon xerogels and their applications on electric double-layer capacitors. J Power Sources 201:340-346. 9. Taylor SJ, Haw MD, Sefcik J, Fletcher AJ (2014) Gelation mechanism of resorcinol-formaldehyde gels investigated by dynamic light scattering. Langmuir 30:10231-10240.

10. Alshrah M, Tran MP, Gong P, Naguib HE, Park CB (2017) Development of high-porosity resorcinol formaldehyde aerogels with enhanced mechanical properties through improved particle necking under 
CO2 supercritical conditions. J Colloid Interface Sci 485:65-74.

11. Rey-Raap N, Menéndez JA, Arenillas A (2013) Optimization of the process variables in the microwave-induced synthesis of carbon xerogels. J Sol-Gel Sci Technol 69:488-497.

12. Calvo EG, Juarez-Perez EJ, Menendez JA, Arenillas A (2011) Fast microwave-assisted synthesis of tailored mesoporous carbon xerogels. J Colloid Interface Sci 357:541-547.

13. Rojas-Cervantes ML (2014) Some strategies to lower the production cost of carbon gels. J Mater Sci 50:1017-1040.

14. Rey-Raap N, Menéndez JA, Arenillas A (2014) Simultaneous adjustment of the main chemical variables to fine-tune the porosity of carbon xerogels. Carbon 78:490-499.

15. Elkhatat AM, Al-Muhtaseb SA (2011) Advances in tailoring resorcinol-formaldehyde organic and carbon gels. Adv Mater 23:2887-2903.

16. Piedboeuf MLC, Léonard AF, Traina K, Job N (2015) Influence of the textural parameters of resorcinol-formaldehyde dry polymers and carbon xerogels on particle sizes upon mechanical milling. Colloids Surf, A 471:124-132.

17. Fairén-Jiménez D, Carrasco-Marín F, Moreno-Castilla C (2006) Porosity and surface area of monolithic carbon aerogels prepared using alkaline carbonates and organic acids as polymerization catalysts. Carbon 44:2301-2307.

18. Job N, Gommes CJ, Pirard R, Pirard JP (2008) Effect of the counter-ion of the basification agent on the pore texture of organic and carbon xerogels. J Non-Cryst Solids 354:4698-4701.

19. Calvo EG, Menéndez JA, Arenillas A (2016) Influence of alkaline compounds on the porosity of resorcinol-formaldehyde xerogels. J Non-Cryst Solids 452:286-290.

20. Brandt R, Fricke J (2004) Acetic-acid-catalyzed and subcritically dried carbon aerogels with a nanometer-sized structure and a wide density range. J Non-Cryst Solids 350:131-135.

21. Barbieri O, Ehrburger-Dolle F, Rieker TP, Pajonk GM, Pinto N, Venkateswara Rao A (2001) Smallangle X-ray scattering of a new series of organic aerogels. J Non-Cryst Solids 285:109-115.

22. Awadallah-F A, Elkhatat AM, Al-Muhtaseb SA (2011) Impact of synthesis conditions on meso- and macropore structures of resorcinol-formaldehyde xerogels. J Mater Sci 46:7760-7769.

23. Resorcinol Based Resins and Applications (2005). In: Resorcinol: Chemistry, Technology and Applications. Springer Berlin Heidelberg, Berlin, Heidelberg, pp 179-261. doi:10.1007/3-540-28090-1_5 24. Rey-Raap N, Arenillas A, Menéndez JA (2016) A visual validation of the combined effect of $\mathrm{pH}$ and dilution on the porosity of carbon xerogels. Microporous and Mesoporous Materials 223:89-93.

25. Lewicki JP, Fox CA, Worsley MA (2015) On the synthesis and structure of resorcinol-formaldehyde polymeric networks - Precursors to 3D-carbon macroassemblies. Polymer (United Kingdom) 69:45-51.

26. Rey-Raap N, Menéndez JA, Arenillas A (2014) RF xerogels with tailored porosity over the entire nanoscale. Microporous and Mesoporous Materials 195:266-275.

27. Alonso-Buenaposada ID, Rey-Raap N, Calvo EG, Menéndez JA, Arenillas A (2015) Effect of methanol content in commercial formaldehyde solutions on the porosity of RF carbon xerogels. Journal of Non-Crystalline Solids 426:13-18.

28. Alonso-Buenaposada ID, Garrido L, Montes-Morán MA, Menéndez JA, Arenillas A (2017) An underrated variable essential for tailoring the structure of xerogel: the methanol content of commercial formaldehyde solutions. J Sol-Gel Sci Technol 83:478-488.

29. Menéndez JA, Phillips J, Xia B, Radovic LR (1996) On the modification and characterization of chemical surface properties of activated carbon: In the search of carbons with stable basic properties. Langmuir 12:4404-4410.

30. Job N, Pirard R, Pirard JP, Alié C (2006) Non intrusive mercury porosimetry: Pyrolysis of resorcinolformaldehyde xerogels. Part Part Syst Charact 23:72-81. 